\title{
Using FT-IR Spectroscopy to Measure Charge Organization in Ionic Liquids
}

\author{
Christopher M. Burba*, Jonathan Janzen, Eric D. Butson ${ }^{\dagger}$, and Gage L. Coltrain \\ Department of Natural Sciences, Northeastern State University, 600 N. Grand Ave., Tahlequah, \\ OK
}

\begin{abstract}
A major goal in the field of ionic liquids is correlating transport property trends with the underlying liquid structure of the compounds, such as the degree of charge organization among the constituent ions. Traditional techniques for experimentally assessing charge organization are specialized and not readily available for routine measurements. This represents a significant roadblock in elucidating these correlations. We use a combination of transmission and polarizedATR infrared spectroscopy to measure the degree of charge organization for ionic liquids. The technique is illustrated with a family of 1-alkyl-3-methylimidazolium trifluoromethansulfonate ionic liquids at $30^{\circ} \mathrm{C}$. As expected, the amount of charge organization decreases as the alkyl side chain is lengthened, highlighting the important role of short-range repulsive interactions in defining quasilattice structure. Inherent limitations of the method are identified and discussed. The quantitative measurements of charge organization are then correlated with trends in the transport properties of the compounds to highlight the relationship between charge and momentum transport and the underlying liquid structure. Most research laboratories possess infrared spectrometers capable of conducting these measurements, thus, the proposed method may represent a costeffective solution for routinely measuring charge organization in ionic liquids.
\end{abstract}

\section{Keywords}

Ionic liquids; charge organization; FT-IR spectroscopy

\section{INTRODUCTION}

\begin{abstract}
Ionic liquids exhibit considerable variation with regard to their transport properties, and understanding the factors leading to these differences constitutes a major area of active research. It is generally recognized that the mechanisms of ion conduction and viscous flow are rooted in the strong cohesive forces that exist among the ions, for these ion-ion interactions lead to long-range, correlated motions of the ions that ultimately define those properties. Thus, understanding the transport properties of ionic liquids hinges on properly understanding the molecular-level structure of the compounds in the liquid phase. There is a growing body of evidence that shows the ions composing an ionic liquid exhibit substantial charge ordering. The primary evidence for charge organization lies in oscillating, out-of-
\end{abstract}

\footnotetext{
*Corresponding author. Tel.: +1 918444 3835; fax: +1 918458 2325. burba@nsuok.edu (C.M. Burba).

$\dagger$ Present Address: Department of Chemistry and Biochemistry, Oklahoma State University, Stillwater, OK.

Author Contributions

The manuscript was written through contributions of all authors. All authors have given approval to the final version of the manuscript.

Supporting Information Available: Instructions for calculating the dipole moment derivative ratio for the ionic liquids investigated.

This material is available free of charge via the Internet at http://pubs.acs.org.
} 
phase cation-anion and cation-cation pair distribution functions obtained from neutron scattering studies of isotopically-labeled materials. ${ }^{1-3}$ Neutron scattering data indicate that charge organization in the liquid state mimics the structure present in the corresponding solid-state crystals. The degree of charge ordering, along with the overall similarity between ion-ion interactions in the liquid and solid phases, becomes less pronounced for large anions $^{1-3}$ and asymmetrical imidazolium cations ${ }^{4-6}$.

Long-range charge ordering is consistent with the quasilattice models that were used to describe ion-ion interactions in high-temperature molten salts. ${ }^{7}$ According to these models, the local environment about the ions is viewed in terms of a perturbed crystal lattice. Ionic liquids with high degrees of charge ordering are expected to possess highly structured quasilattices that resemble the solid-state crystal structures of the compounds. Although the degree of quasilattice structure present in an ionic liquid can be experimentally determined, the techniques for doing so (e.g., neutron scattering) are highly specialized and generally limited to a handful of facilities around the world. It is impractical to routinely measure quasilattice structure for the large number of ionic liquids that currently exist or are synthetically feasible with the currently available techniques.

Burba and Frech ${ }^{8}$ proposed a solution to this problem by quantifying quasilattice structure with simple FT-IR spectroscopic experiments. The heart of the approach is to compare two independent measurements of the dipole moment derivative for select vibrational modes of the ions composing the ionic liquid. The first measurement evaluates the dipole moment derivative of a normal mode from transmission FT-IR spectra of the ionic liquid using dipolar coupling theory. This approach assumes that the constituent ions occupy locations within a perfectly organized quasilattice. Measuring the optical constants of the ionic liquid from polarized ATR FT-IR spectra provides the second assessment of the dipole moment derivative for the same vibrational mode. This measurement makes no assumptions about the long-range organization of the ions within the liquid. Therefore, comparing the dipole moment derivative estimates can test the robustness of the core assumptions of dipolar coupling theory in the context of ionic liquids. Namely, is a highly structured quasilattice a good model for the liquid structure of a particular ionic liquid?

The purpose of this article is to demonstrate how quasilattice structure can be obtained from infrared spectroscopy and understand some of the limitations associated with quantifying charge organization with this methodology. The 1-alkyl-3-methylimidazolium

trifluoromethanesulfonate family of ionic liquids, $\left[\mathrm{C}_{n}\right.$ mim $] \mathrm{CF}_{3} \mathrm{SO}_{3}$ with $n=2,4,6$, and 8 , is used to illustrate the overall strategy. We chose this class of ionic liquids for two reasons. First, the symmetric stretching motion of the $\mathrm{SO}_{3}$ portion of the $\mathrm{CF}_{3} \mathrm{SO}_{3}{ }^{-}$anion, $\mathrm{v}_{\mathrm{S}}\left(\mathrm{SO}_{3}\right)$, is a non-degenerate, strong band that is reasonably well-separated from IR bands of the imidazolium cations. Non-degenerate vibrational modes are particularly useful, for the lack of band splitting due to disruption of the point group symmetry of the ion greatly simplifies the analysis of the spectral data. Second, the liquid structure of triflate-containing ionic liquids has been extensively studied through molecular dynamics (MD) simulations. ${ }^{9-11}$ Thus, these materials are particularly well suited to test the validity of quantifying charge organization in ionic liquids with infrared spectroscopy. Quantitative measurements of charge organization are also correlated with the transport properties of the materials to highlight the relationship between the transport properties of an ionic liquid and its liquid structure. 


\section{EXPERIMENTAL METHODS}

\section{Samples Investigated}

1-Ethyl-3-methylimidazolium trifluoromethanesulfonate $\left(\left[\mathrm{C}_{2} \mathrm{mim}\right] \mathrm{CF}_{3} \mathrm{SO}_{3}\right), 1$-butyl-3ethylimidazolium trifluoromethanesulfonate $\left(\left[\mathrm{C}_{4} \mathrm{mim}\right] \mathrm{CF}_{3} \mathrm{SO}_{3}\right)$, 1-ethyl-3hexylimidazolium trifluoromethanesulfonate $\left(\left[\mathrm{C}_{6} \mathrm{mim}\right] \mathrm{CF}_{3} \mathrm{SO}_{3}\right)$, and 1-ethyl-3octylimidazolium trifluoromethanesulfonate $\left(\left[\mathrm{C}_{8} \mathrm{mim}\right] \mathrm{CF}_{3} \mathrm{SO}_{3}\right)$ ionic liquids were purchased from Iolitec and used without further purification. All compounds were stored in a dry environment.

\section{Density and Transport Property Measurements}

Vercher et al. ${ }^{12}$ reported temperature-dependent density data for $\left[\mathrm{C}_{2} \mathrm{mim}\right] \mathrm{CF}_{3} \mathrm{SO}_{3}$, and the value of the density for this ionic liquid at $30^{\circ} \mathrm{C}$ is interpolated from their data. Density measurements of $\left[\mathrm{C}_{4} \mathrm{mim}\right] \mathrm{CF}_{3} \mathrm{SO}_{3},\left[\mathrm{C}_{6} \mathrm{mim}\right] \mathrm{CF}_{3} \mathrm{SO}_{3}$, and $\left[\mathrm{C}_{8} \mathrm{mim}\right] \mathrm{CF}_{3} \mathrm{SO}_{3}$ are obtained with an Anton Paar DMA density meter. Temperature-dependent ionic conductivities are collected with an Accumet $\mathrm{AB} 30$ conductance meter using a 4-wire conductivity probe with a nominal cell constant of $10 \mathrm{~cm}^{-1}$. Calibration of the conductivity probe was achieved with a $0.20 \mathrm{M} \mathrm{KCl}$ aqueous solution of known conductivity. A Brookfield LV-DVE viscometer, containing a small-sample adapter (SSA-18), is used to measure temperature-dependent viscosity data.

\section{Infrared Spectroscopic Measurements}

Transmission FT-IR spectra are recorded by sandwiching small quantities of ionic liquid between two ZnSe windows. FT-IR spectra are collected with a Nicolet 6700 FT-IR spectrometer, operating with $1 \mathrm{~cm}^{-1}$ resolution and under a dry-air purge. The spectrometer is equipped with a DTGS detector. The IR beam path is restricted to a diameter of $10 \mathrm{~mm}$ at the sample to ensure that the edges of the $\mathrm{ZnSe}$ windows, where the amount of ionic liquid often becomes depleted, are eliminated from analysis. Sample thicknesses are adjusted by controlling the total volume deposited on the windows to prevent power saturation broadening of the IR bands. Volumes of $\sim 0.8 \mu \mathrm{L}$ generally resulted in acceptable spectra with absorbance values near one for the $\mathrm{v}_{\mathrm{s}}\left(\mathrm{SO}_{3}\right)$ band. The distance between spectral fringes over the range $4000-5000 \mathrm{~cm}^{-1}$ are used to measure sample thicknesses as this region of the spectrum is free from vibrational modes of the samples. Most samples have thicknesses near $8 \mu \mathrm{m}$.

Attenuated total reflection (ATR) FT-IR spectra are measured with a PIKE VeeMaxII variable-angle ATR attachment for the Nicolet 6700 FT-IR spectrometer. Samples are placed on a germanium ATR crystal, which has a $45^{\circ}$ angle of incidence, and spectra are recorded with $1 \mathrm{~cm}^{-1}$ resolution under a dry air purge. A wire grid polarizer is placed in the IR beam path to obtain $s$-polarized ATR FT-IR spectra.

\section{Theoretical Framework for Measuring Quasilattice Structure}

The degree of quasilattice structure present in an ionic liquid can be determined by comparing independent measurements of the dipole moment derivative for a particular vibrational mode. For the sake of completeness, we briefly review the key equations involved in calculating the dipole moment derivatives as well as the core theoretical assumptions that enable us to quantitatively measure relative differences in quasilattice structure for ionic liquids. We begin with dipolar coupling theory, which is used to derive dipole moment derivatives from transmission FT-IR spectra of the ionic liquids. Afterwards, we consider the modified Kramers-Kronig transform that allows dipole moment derivatives to be calculated from polarized ATR FT-IR spectra. 
During a typical transmission IR experiment, incident electromagnetic radiation is partly transmitted through the sample, with the remaining light being either absorbed or reflected. Collective lattice-like coupling of ions in a quasilattice can produce long-wavelength vibrational modes in the sample and result in optical dispersion. The electromagnetic field of the vibrating charges in a normal mode may couple with the mechanical vibrational motion of the mode, resulting in a polariton that is neither purely electromagnetic nor purely mechanical in nature. Such a polariton does not support the propagation of a photon through the quasilattice. Thus, a substantial amount of reflection may be expected during a transmission FT-IR experiment for intense bands between frequencies corresponding to the transverse optic (TO) and longitudinal optic (LO) modes. ${ }^{13}$

Dipolar coupling theory ${ }^{14}$ has been previously used to relate the magnitude of the TO-LO splitting to the dipole moment derivatives of vibrational modes in a variety of isotropic and anisotropic crystals ${ }^{15-17}$, disordered salts ${ }^{18,19}$, and molten salts ${ }^{8,19}$. A simplified form of dipolar coupling theory results in the following expression for the TO-LO splitting of a normal mode in a unimolecular unit cell: ${ }^{16}$

$$
\nu_{L O}^{2}-\nu_{T O}^{2}=4 \pi N\left(\frac{\partial \mu}{\partial q}\right)^{2}
$$

Here $v_{L O}$ and $v_{T O}$ are the frequencies of the LO and TO modes, respectively, $N$ is the dipole density, and $|(\partial \mu / \partial q)|$ is the dipole moment derivative of a vibrational mode. Therefore, measurements of TO and LO band frequencies from a transmission FT-IR experiment may be used to estimate the dipole moment derivative for a vibrational mode of a cation or anion in an ionic liquid. It is important to recognize, however, the application of Eq. (1) implicitly assumes a perfectly ordered lattice with a single formula unit of the ionic liquid occupying the unit cell. This assumption is certainly violated to some extent for ionic liquids in the liquid phase.

An alternative estimate of the dipole moment derivative for a vibrational mode is available, which relies neither on the quasilattice model nor dipolar coupling theory. Bardwell and Dignam ${ }^{20-22}$ developed a routine method for determining the optical constants of a compound from single-reflection polarized ATR FT-IR spectroscopy. The algorithm is based on a Kramers-Kronig transform between $\ln \left(\sqrt{R_{s}(\nu)}\right)$, where $R_{S}(v)$ is the reflectance in an $s$-polarized ATR FT-IR experiment, and the phase angle, $\delta_{s}(v)$, of the complex Fresnel reflection coefficient:

$$
\delta_{s}(\nu)=2 \arctan \left(\frac{\sqrt{n_{o}^{2} \sin ^{2} \theta_{o}-n_{v i s}^{2}}}{n_{o} \cos \theta_{o}}\right)+\frac{2}{\pi} \int_{0}^{\infty} \frac{\nu \ln \left(\sqrt{R_{s}(\nu)}\right)}{\nu_{a}^{2}-\nu^{2}} d \nu_{a} .
$$

Here, $n_{o}$ is the refractive index for the ATR crystal, $\theta_{o}$ is the angle of incidence, and $n_{V i S}$ is the refractive index of the ionic liquid measured in the visible region of the electromagnetic spectrum. The Cauchy principle value of the integral is required to evaluate Eq. (2). The complex Fresnel coefficient, $r(v)$, is given as

$$
\widehat{r}(\nu)=\sqrt{R_{s}(\nu)} \exp \left(i \delta_{s}(\nu)\right) .
$$


Refractive indices, $n(v)$, and extinction coefficients, $k(v)$, of the ionic liquid may then be directly calculated from complex Fresnel coefficient with the following two equations:

$$
\begin{aligned}
& \left.n(\nu)=n_{o} \operatorname{Re}\left[\sin ^{2} \theta_{o}+\left(\frac{1-\widehat{r}_{s}(\nu)}{1+\widehat{r}_{s}(\nu)}\right)^{2} \cos ^{2} \theta_{o}\right]^{1 / 2}, \quad \text { (4a }\right) \\
& k(\nu)=-n_{o} \operatorname{Im}\left[\sin ^{2} \theta_{o}+\left(\frac{1-\widehat{r}_{s}(\nu)}{1+\widehat{r}_{s}(\nu)}\right)^{2} \cos ^{2} \theta_{o}\right]^{1 / 2},
\end{aligned}
$$

The dipole moment derivative for a particular vibrational mode may be determined by integrating the extinction coefficient data, ${ }^{8}$

$$
\left(\frac{\partial \mu}{\partial q}\right)^{2}=\frac{12 c \nu_{o}}{N} \int_{b a n d} k(\nu) d \nu
$$

These two approaches provide independent measurements of the dipole moment derivative for a particular vibrational mode of an ionic liquid. The crucial difference is that dipolar coupling theory assumes a perfect crystalline lattice, whereas the method proposed by Bardwell and Dignam do not rely on this assumption. Thus, comparing the two assessments should give insight into the degree that the core assumption of dipolar coupling theory is violated.

The dipole moment derivative ratio is a useful means for testing the robustness of the assumptions for dipolar coupling theory, and thereby quantifying quasilattice structure, for ionic liquids. Dipole moment derivative ratios are calculated by dividing the dipole moment derivative derived from dipolar coupling theory, $\left|(\partial \mu / \partial q)_{D C T}\right|$, by the dipole moment derivative obtained from the Kramers-Kronig transform, $\mid(\partial \mu / \partial q)_{K K T}$. Ionic liquids with highly structured quasilattices should better satisfy the assumptions of dipolar coupling theory than those with loosely structured quasilattices. In the former case, the two measurements of the dipole moment derivative should be similar, and the ratio of the two estimates should approach a value of one. In contrast, ionic liquids with poorly structured quasilattices should show a marked disparity in the two estimates with $\left|(\partial \mu / \partial q)_{D C T}\right|<\mid(\partial \mu /$ $\partial q)_{K K T}$. In this case, the dipole moment derivative ratio will approach zero. Detailed instructions for calculating the dipole moment derivative ratio are provided as Supporting Information.

\section{RESULTS AND DISCUSSION}

\section{Assessing Quasilattice Structure for $\left[\mathrm{C}_{n} \mathrm{mim}\right] \mathrm{CF}_{3} \mathrm{SO}_{3}$ lonic Liquids}

Transmission FT-IR spectra of $\left[\mathrm{C}_{n} \mathrm{mim}\right] \mathrm{CF}_{3} \mathrm{SO}_{3}$ are presented in Fig. 1A. There is noticeable asymmetry in the bands, which gradually decreases as the number of carbon atoms in the alkyl side chain increases. Spectral deconvolution results in three overlapped bands for $\left[\mathrm{C}_{2} \mathrm{mim}\right] \mathrm{CF}_{3} \mathrm{SO}_{3}$, and the curve fitting results are shown in Figure 1B. The two dominant bands are assigned to the $\mathrm{LO}$ and $\mathrm{TO}$ modes of $\mathrm{V}_{\mathrm{s}}\left(\mathrm{SO}_{3}\right)$. A weak band near 1020 $\mathrm{cm}^{-1}$ is also observed in the IR spectra; this band is assigned to in-plane ring stretching of the imidazolium cations. ${ }^{23}$ The degree of TO-LO band splitting-as calculated from a critical point analysis (see the Supporting Information for details) — decreases as the length of the alkyl side chain increases and becomes very small for $\left[\mathrm{C}_{6} \mathrm{mim}\right] \mathrm{CF}_{3} \mathrm{SO}_{3}$ and $\left[\mathrm{C}_{8} \mathrm{mim}\right] \mathrm{CF}_{3} \mathrm{SO}_{3}$. 
Dipole moment derivative ratios are obtained for each of the ionic liquids and plotted as a function of the number of carbon atoms composing the alkyl side-chain (Fig. 2). All four ionic liquids have small dipole moment derivative ratios. This suggests that the fundamental assumptions undergirding dipolar coupling theory, especially the requirement of a perfectly organized crystal structure, are poorly satisfied by these compounds. Consequently, the degree of quasilattice structure is low for these materials and likely does not persist for more than a few solvation shells.

Among the four ionic liquids studied, the highest amount of quasilattice structure was observed for the ionic liquid with the shortest alkyl chain length. Gabl and coworkers 9 extensively investigated the liquid structure of $\left[\mathrm{C}_{2} \mathrm{mim}\right] \mathrm{CF}_{3} \mathrm{SO}_{3}$ through $\mathrm{MD}$ simulations on an ensemble of $2000\left[\mathrm{C}_{2} \mathrm{mim}\right]^{+}$and $\mathrm{CF}_{3} \mathrm{SO}_{3}{ }^{-}$ion pairs. It is worthwhile to consider the results of those simulations to provide a context for interpreting our measurements of quasilattice structure obtained through dipole moment derivative ratios. As discussed earlier, cation-cation and cation-anion radial distribution functions are commonly used to assess charge organization in ionic liquids. Calculated $\left[\mathrm{C}_{2} \mathrm{mim}\right]^{+}-\left[\mathrm{C}_{2} \mathrm{mim}\right]^{+}$and $\left[\mathrm{C}_{2} \mathrm{mim}\right]^{+}-$ $\mathrm{CF}_{3} \mathrm{SO}_{3}{ }^{-}$center-of-mass radial distribution functions from the MD simulations are out of phase with one another and persist to $40 \AA$ from the central cation. Furthermore, orientational correlation functions for the dipole moments of the cations and anions were evaluated, and the $\mathrm{CF}_{3} \mathrm{SO}_{3}{ }^{-}$anions exhibit pronounced antiparallel alignment of the molecular axes, leading to an asymptotic value of 0.522 for the anion-anion Kirkwood Gfactor. In contrast, cations show little orientational ordering with a reported cation-cation Kirkwood G-factor near one. Differences between the mutual orientational ordering of the cations and anions are attributed to the lower charge density of the cations relative to the anions.

Although substantial charge ordering is reported in the MD simulations, the immediate coordination environment about the ions (i.e., the ions composing the coordination cage for a particular ion) is not solely populated by counterions. ${ }^{10}$ Indeed, there is an average of ten cationic and eight anionic neighbors surrounding a given $\left[\mathrm{C}_{2} \mathrm{mim}\right]^{+}$ion, whereas approximately eight cationic and four anionic neighbors compose the ion cage of a $\mathrm{CF}_{3} \mathrm{SO}_{3}{ }^{-}$ anion. The combination of high coordination numbers and long ion-residence times led Schröder ${ }^{10}$ to conclude that $\left[\mathrm{C}_{2}\right.$ mim $] \mathrm{CF}_{3} \mathrm{SO}_{3}$ possesses long-lived ion cages instead of longlived ion pairs; an observation that is consistent with the presence of a single $\delta_{5}\left(\mathrm{CF}_{3}\right)$ band in the Raman spectrum of the ionic liquid. ${ }^{24}$

Neutron scattering experiments ${ }^{4-6}$ of ionic liquids possessing asymmetric cations suggest that the number of carbon atoms present in the alkyl side chain is correlated with smaller amounts of charge organization. Dipole moment derivative ratios linearly decrease as the length of the alkyl side chain increases from $\mathrm{C}_{2}$ to $\mathrm{C}_{6}$ (Fig. 2). Smaller values of the dipole moment derivative ratios indicate less quasilattice structure for ionic liquids with long side chains compared to shorter chain analogs. This observation is consistent with the prior experimental and computational data concerning the relationship between charge organization and side chain length, thereby increasing confidence in our quantitative assessments of the liquid structure of these materials.

The dipole moment derivative ratio is slightly higher for $\left[\mathrm{C}_{8} \mathrm{mim}\right] \mathrm{CF}_{3} \mathrm{SO}_{3}$ than $\left[\mathrm{C}_{6} \mathrm{mim}\right] \mathrm{CF}_{3} \mathrm{SO}_{3}$. The $\mathrm{v}_{\mathrm{s}}\left(\mathrm{SO}_{3}\right)$ line shape is relatively symmetric for this compound, which implies the TO and LO band splitting is very small. In the limit of zero TO-LO splitting, the critical point analysis used to extract the TO and LO mode frequencies will overestimate the frequency of the $\mathrm{LO}$ mode. The combination of an overestimated $\mathrm{LO}$ frequency and a lower dipole density for $\left[\mathrm{C}_{8}\right.$ mim $] \mathrm{CF}_{3} \mathrm{SO}_{3}$ compared to the other ionic liquids artificially increases | $(\partial \mu / \partial q)_{D C T} \mid$ and, consequently, the dipole moment derivative ratio for this compound. Most 
likely $\left[\mathrm{C}_{8} \mathrm{mim}\right] \mathrm{CF}_{3} \mathrm{SO}_{3}$ exhibits similar degrees of charge organization as $\left[\mathrm{C}_{6} \mathrm{mim}\right] \mathrm{CF}_{3} \mathrm{SO}_{3}$, and both compounds have very low amounts of charge organization. The foregoing analysis of $\left[\mathrm{C}_{8} \mathrm{mim}\right] \mathrm{CF}_{3} \mathrm{SO}_{3}$ is an excellent illustration of the inherent limitations in using dipole moment derivative ratios calculated with the critical point values of the IR spectrum to characterize ionic liquids with very small amounts of charge organization.

\section{Factors that Influence Quasilattice Structure for Ionic Liquids}

Ionic liquids are composed entirely of ions, and the distances between neighboring ions are relatively short compared to the distances separating ions in conventional solutions. The potential energy environment of an individual ion is defined by the cumulative Coulombic interactions between the ion and other cations and anions occupying sites within the quasilattice. Indeed, one can envision a quasilattice analog to the lattice energy of an ionic solid-state material. For crystalline compounds, the lattice energy is often calculated with the Born-Landé equation, ${ }^{25}$

$$
V\left(r_{o}\right)=-\frac{M z_{+}\left|z_{-}\right| e^{2}}{4 \pi \varepsilon_{o} r_{o}}\left(1-\frac{1}{n}\right)
$$

where $M$ is the Madelung constant, $z$ is the charge on each ion, $e$ is the elementary charge, $\epsilon_{o}$ is the permittivity of free space, $r_{o}$ is the equilibrium ion-ion distance in the crystal, and $n$ is the Born exponent. Conditions of charge neutrality and packing effects among ions organize cations and anions of an ionic liquid into a quasilattice in the immediate vicinity about any particular ion. Thus, the local environment about the ion will resemble an ionic solid. However, the translational symmetry of the quasilattice begins to break down as the distance from the ion increases. Consequently, the meaning of a Madelung constant begins to lose significance for these materials, for the summation inherent in determining the Madelung constant cannot be extended to encompass the entire ionic liquid.

Hansen and McDonald ${ }^{26}$ review a number of models that have been proposed to describe the liquid structure of molten salts. Rigid-ion models, which ignore ion polarizabilities and assume the interactions among ions are pair-wise additive, are among the simpler models available. According to this formalism, the potential energy is written as the sum of shortrange repulsion terms, $V_{N}^{S}\left(\mathbf{r}^{N}\right)$, and Coulombic interactions,

$$
V_{N}\left(\mathbf{r}^{N}\right)=V_{N}^{S}\left(\mathbf{r}^{N}\right)+\sum_{i=1}^{N} \sum_{j>i}^{N} \frac{z_{j} z_{i} e^{2}}{\left|\mathbf{r}_{j}-\mathbf{r}_{i}\right|}
$$

Short-range interactions among the ions are often modeled with soft-core potentials that include a single length parameter set equal to the equilibrium ion-ion separation distance. Together the soft-core repulsion and Coulombic interaction terms define the potential energy function that is used to model simple molten salts.

Long alkyl side chains on the imidazolium cations will frustrate local packing of the ions, resulting in larger equilibrium ion-ion distances. In the context of Eq. (7), larger equilibrium ion-ion distances increase the magnitude of the short-range repulsion term. This has the net effect of destabilizing the quasilattice and reducing the amount of charge ordering present in the ionic liquid. Consequently, there is a negative correlation between the dipole moment derivative ratio and the number of carbon atoms present in the side chain as observed in Fig. 2 . 
$\mathrm{X}$-ray scattering studies of imidazolium ionic liquids suggest there is some degree of nanophase segregation that is driven by the interaction energies of the non-polar side chains and the polar imidazolium rings. ${ }^{27-31}$ These interactions become significant for ionic liquids with long side chains. Although X-ray scattering studies of the $\left[\mathrm{C}_{n} \mathrm{mim}\right] \mathrm{CF}_{3} \mathrm{SO}_{3}$ ionic liquids were not performed, it is likely that $\left[\mathrm{C}_{6} \mathrm{mim}\right] \mathrm{CF}_{3} \mathrm{SO}_{3}$ and $\left[\mathrm{C}_{8} \mathrm{mim}\right] \mathrm{CF}_{3} \mathrm{SO}_{3}$ experience some degree of nano-phase segregation. Undoubtedly, the polar and non-polar interactions among the imidazolium ions will begin to play a significant role in defining the liquid structure of ionic liquids, and this may also explain, at least in part, why those ionic liquids exhibit lower amounts of quasilattice structure as measured through the dipole moment derivative ratio.

\section{Correlation between Quasilattice Structure and lonic Liquid Transport Properties}

A key component to understanding the mechanisms of ion conduction and viscous flow is having an accurate picture of the potential energy environment the ions experience, for this defines the potential energy barriers that ions must overcome to migrate between sites in the ionic liquid. The competition between local packing effects and Coulombic interactions defines the potential energy of the ions, organizing them onto the sites of a quasilattice. Therefore, one may expect a relationship between quasilattice structure and the transport properties of the ionic liquids since both are influenced by the same inter-ionic forces that exist among the ions.

A useful means for displaying trends in the molar conductivity $(\Lambda)$ and fluidity $\left(\mathrm{n}^{-1}\right)$ is achieved with a Walden plot of $\log \Lambda$ vs $\log \eta^{-1.7}$ Molecular solvents generally appear near the lower right corner of a Walden plot, whereas ion-conducting glasses occupy the upper left corner. The mechanism of ion conduction for an ideal system is believed to be coupled to the same long-range correlated motions that govern viscous flow. ${ }^{32}$ Hence, the transport properties of ideal systems fall on or near the $\log \Lambda=\log \eta^{-1}$ line; dilute $\mathrm{KCl}$ aqueous solutions are common examples of ideal systems because those solutions consist of fully dissociated ions. Although such formalism is often used, it is not immediately clear what the liquid structure is for "ideal" ionic liquids. Regardless, the location of an ionic liquid on a Walden plot is believed to be related in some way to the quasilattice structure of the compound, with increased amounts of quasilattice structure corresponding to compounds that are closer to the ideal system line. ${ }^{32}$

A Walden plot for $\left[\mathrm{C}_{n} \mathrm{mim}\right] \mathrm{CF}_{3} \mathrm{SO}_{3}$ is depicted in Fig. 3. These ionic liquids possess transport properties at $T=30^{\circ} \mathrm{C}$ that place them near the ideal system line, with increased deviation from ideal system behavior as the alkyl side chain is lengthened. Molar conductivities and fluidities for $\left[\mathrm{C}_{2} \mathrm{mim}\right] \mathrm{CF}_{3} \mathrm{SO}_{3},\left[\mathrm{C}_{4} \mathrm{mim}\right] \mathrm{CF}_{3} \mathrm{SO}_{3}$, and $\left[\mathrm{C}_{6} \mathrm{mim}\right] \mathrm{CF}_{3} \mathrm{SO}_{3}$ are plotted as a function of their dipole moment derivative ratios in Fig. 4. Data for $\left[\mathrm{C}_{8} \mathrm{mim}\right] \mathrm{CF}_{3} \mathrm{SO}_{3}$ are not included since the dipole moment derivative ratio is unreliable (see discussion above). Both transport properties are linearly correlated with the dipole moment derivative ratio. Ions occupying sites within the quasilattice will undergo translatory vibrational motion within a cage defined by the nearest neighbor ions. Charge transport occurs when the translatory vibrational motion of the ion transitions to translational motion by overcoming the potential energy barrier that confines the ion to its quasilattice site. By way of comparison, momentum transport occurs when layers of ions slide past each another in response to a shear stress field. This scenario differs from ion conduction in that multiple ions undergo cooperative movement by overcoming the potential energy barriers that bind the ions to their quasilattice sites. As argued in previous section, steric interactions that arise from longer alkyl side chains attached to the imidazolium rings decrease quasilattice structure by increasing the short-range repulsion interactions between the ions. Larger cation sizes are also expected to result in lower cation mobilities (smaller molar conductivities) and lower fluidities. Thus, it is plausible that trends in the liquid structure and the transport 
properties of the 1-alkyl-3-methylimidazolium trifluoromethanesulfonate ionic liquids are both controlled by differences in the relative sizes of the imidazolium cations and the corresponding changes in the potential energy environments of the ions due to these differences.

\section{CONCLUSIONS}

Unraveling the complex relationship between molecular-level structure and the macroscopic properties of ionic liquids requires quantitative information about the long-range ion-ion interactions present in ionic liquids. General trends will only be discovered by examining correlations between charge organization and ionic liquid transport properties for a large number of systems. However, the needed data sets will be difficult to obtain using traditional methods of measuring liquid structure because the techniques are highly specialized. In this article, we use a combination of IR spectroscopic techniques to characterize the degree of charge organization present in a family of imidazolium-based ionic liquids. Longer alkyl side chains grafted onto the imidazolium ions increase the shortrange repulsive interactions among the ions and degrade charge organization, consistent with expectations from prior studies on these materials. The assessments of charge organization were then used explain relative differences in the transport properties of the materials.

Most research laboratories possess infrared spectrometers capable of conducting these infrared spectroscopic measurements; hence, the proposed methodology represents a widespread, cost-effective solution to the need for routine assessments of charge organization. We believe the availability of experimental measurements of charge organization for a wide assortment of ionic liquids will facilitate deeper understanding of the correlation between the transport properties of the materials and their underlying liquid structure. Such information will be especially valuable for the targeted design of ionic liquids with improved functionality in specific market niches.

\section{Supplementary Material}

Refer to Web version on PubMed Central for supplementary material.

\section{Acknowledgments}

Funding Sources

This project (or publication) was supported by the National Institute of General Medical Sciences of the National Institutes of Health through Grant Number 8P20GM103447 and the Oklahoma State Regents for Higher Education.

The authors thank Dr. Matt Petrowsky and Dr. Roger Frech for providing temperature-dependent density data.

\section{References}

1. Hardacre C, Holbrey JD, McMath SEJ, Bowron DT, Soper AK. Structure of molten 1,3dimethylimidazolium chloride using neutron diffraction. J Chem Phys. 2003; 118:273.

2. Hardacre C, McMath SEJ, Nieuwenhuyzen M, Bowron DT, Soper AK. Liquid structure of 1,3dimethylimidazolium salts. J Phys : Condens Matter. 2003; 15:S159.

3. Deetlefs M, Hardacre C, Nieuwenhuyzen M, Padua AAH, Sheppard O, Soper AK. Liquid structure of the ionic liquid 1,3-dimethylimidazolium bis $\{$ (trifluoromethyl)sulfonyl $\}$ amide. J Phys Chem B. 2006; 110:12055. [PubMed: 16800516]

4. Hardacre C, Holbrey JD, Mullan CL, Youngs TGA, Bowron DT. Small angle neutron scattering from 1-alkyl-3-methylimidazolium hexafluorophosphate ionic liquids $\left(\left[\mathrm{C}_{n} \mathrm{mim}\right]\left[\mathrm{PF}_{6}\right], n=4,6\right.$, and 8). J Chem Phys. 2010; 133:074510. [PubMed: 20726655] 
5. Fujii K, Soejima Y, Kyoshoin Y, Fukuda S, Kanzaki R, Umebayahi Y, Yamaguchi T, Ishiguro SI, Takamuku T. Liquid structure of room-temperature ionic liquid, 1-ethyl-3-methylimidazolium bis(trifluoromethanesulfonyl) imide. J Phys Chem B. 2008; 112:4329. [PubMed: 18348563]

6. Triolo A, Mandanici A, Russina O, Rodriguez-Mora V, Cutroni M, Hardacre C, Nieuwenhuyzen M, Bleif H-J, Keller L, Ramos MA. Thermodynamics, structure, and dynamics in room temperature ionic liquids: The case of 1-butyl-3-methylimidazolium hexafluorophosphate ([bmim] $\left.\left[\mathrm{PF}_{6}\right]\right)$. J Phys Chem B. 2006:110.

7. Xu W, Cooper EI, Angell CA. Ionic liquids: Ion mobilities, glass temperatures, and fragilities. J Phys Chem B. 2003; 107:6170.

8. Burba CM, Frech R. Existence of optical phonons in the room temperature ionic liquid 1-ethyl-3methylimidazolium trifluoromethanesulfonate. J Chem Phys. 2011; 134:134503. [PubMed: 21476760]

9. Gabl S, Schröder C, Steinhauser O. Computational studies of ionic liquids: Size does matter and time too. J Chem Phys. 2012; 137:094501. [PubMed: 22957575]

10. Schröder C. Collective translational motions and cage relaxations in molecular ionic liquids. J Chem Phys. 2011; 135:024502. [PubMed: 21766952]

11. Schröder C, Sonnleitner T, Buchner R, Steinhauser O. The influence of polarizability on the dielectric spectrum of the ionic liquid 1-ethyl-3-methylimidazolium triflate. Phys Chem Chem Phys. 2011; 13:12240. [PubMed: 21643580]

12. Vercher E, Llopis FJ, González-Alfaro MV, Martínez-Andreu A. Density, speed of sound, and refractive index of 1-ethyl-3-methylimidazolium trifluoromethanesulfonate with acetone, methyl acetate, and ethyl acetate at temperatures from (278.15 to 328.15) K. J Chem Eng Data. 2010; 55:1377.

13. Sherwood, PMA. Vibrational Spectroscopy of Solids. Cambridge University Press; Cambridge: 1972.

14. Decius JC. Dipolar coupling and molecular vibration in crystals. I. General theory. J Chem Phys. 1968; 49:1387.

15. Frech R. Spectroscopic properties of the orthoborate ion in an indium borate crystal. J Chem Phys. 1974; 60:1678.

16. Frech R, Decius JC. Dipolar coupling and molecular vibrations in crystals. IV. Frequency shifts and dipole moment derivatives. J Chem Phys. 1971; 54:2374.

17. Wu GJ, Frech R. The optical and spectroscopic properties of the sulfate ion in various crystalline environments. J Phys Chem. 1977; 66:1352.

18. Devlin JP, James DW, Frech R. Correlation field splitting of vibrational modes in disordered sodium nitrate. I. Chem Phys Lett. 1972; 12:602.

19. Devlin JP, Pollard G, Frech R. ATR [attenuated total reflectance] infrared spectra of uniaxial nitrate crystals. J Chem Phys. 1970; 53:4147.

20. Bardwell JA, Dignam MJ. Routine determination of the absorption and dispersion spectra of solids with a Fourier-transform infrared spectrometer. Anal Chim Acta. 1985; 172:101.

21. Bardwell JA, Dignam MJ. Extensions of the Kramers-Kronig transformation that cover a wide range of practical spectrocopic applications. J Chem Phys. 1985; 83:5468.

22. Bardwell JA, Dignam MJ. Routine method for the determination of the optical constants of liquids. Anal Chim Acta. 1986; 181:253.

23. Talaty ER, Raja S, Storhaug VJ, Dölle A, Carper WR. Raman and infrared spectra and ab Initio calculations of $\mathrm{C}_{2-4}$ mim imidazolium hexafluorophosphate ionic liquids. J Phys Chem B. 2004; 108:13177.

24. Burba CM, Rocher NM, Frech R. Hydrogen-bonding and ion-ion interactions in solutions of triflic acid and 1-ethyl-3-methylimidazolium triflate. J Phys Chem B. 2009; 113:11453. [PubMed: 19637847]

25. Huheey, JE.; Keiter, EA.; Keiter, RL. Inorganic Chemistry: Principles of Structure and Reactivity. 4. Harper Collins; 1993.

26. Hansen, J-P.; McDonald, IR. Theory of Simple Liquids. 3. Elsevier Academic Press; London: 2006. 
27. Russina O, Triolo A. New experimental evidence supporting the mesoscopic segregation model in room temperature ionic liquids. Faraday Discuss. 2012; 154:97. [PubMed: 22455016]

28. Russina O, Triolo A, Gontrani L, Caminiti R. Mesoscopic Structural Heterogeneities in RoomTemperature Ionic Liquids. J Phys Chem Lett. 2012; 3:27.

29. Triolo A, Russina O, Bleif HJ, Di Cola E. Nanoscale segregation in room temperature ionic liquids. J Phys Chem B. 2007; 111:4641. [PubMed: 17388414]

30. Triolo A, Russina O, Fazio B, Triolo R, Di Cola E. Morphology of 1-alkyl-3-methylimidazolium hexafluorophosphate room temperature ionic liquids. Chem Phys Lett. 2008; 457:362.

31. Annapureddy HVR, Kashyap HK, De Biase PM, Margulis CJ. What is the origin of the prepeak in the x-ray scattering of imidazolium-based room-temperature ionic liquids? J Phys Chem B. 2010; 114:16838. [PubMed: 21077649]

32. Angell CA, Ansari Y, Zhao Z. Ionic liquids: Past, present and future. Faraday Discuss. 2011; 154:9. [PubMed: 22455011] 


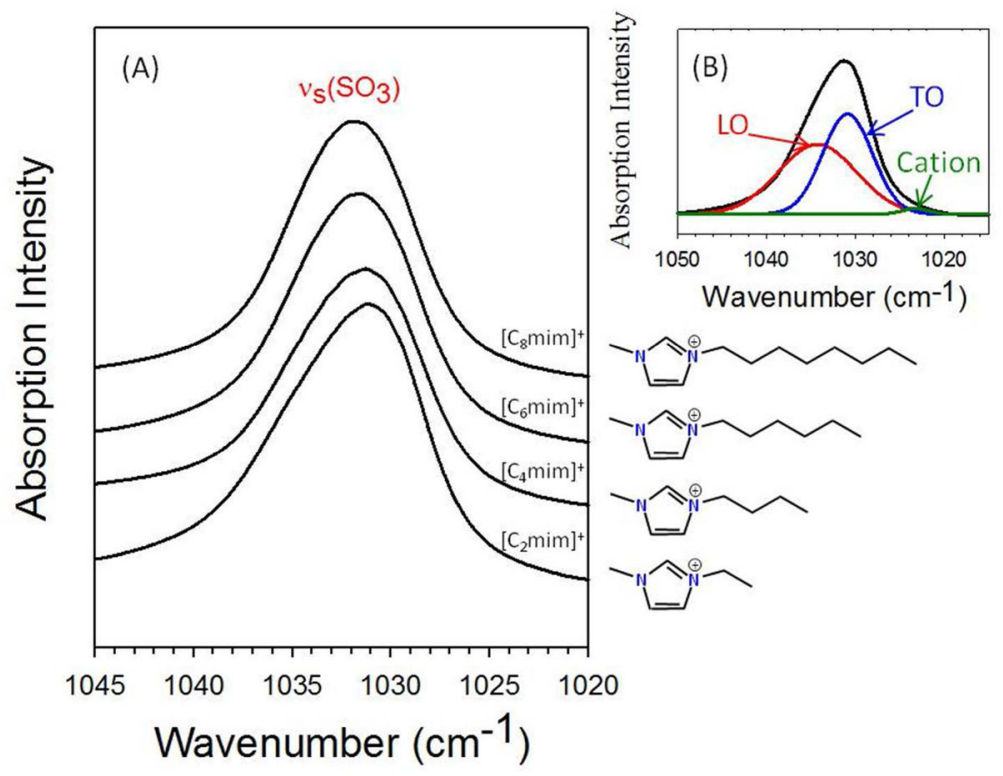

Fig. 1.

(A) Transmission FT-IR spectra of $\left[\mathrm{C}_{n} \mathrm{mim}\right] \mathrm{CF}_{3} \mathrm{SO}_{3}$ are recorded at room temperature. Spectral deconvolution reveals three overlapped bands between 1045 and $1020 \mathrm{~cm}^{-1}$. Two bands are assigned to the LO and $\mathrm{TO} \mathrm{v}_{\mathrm{s}}\left(\mathrm{SO}_{3}\right)$ bands, while the third band is attributed to a cation vibrational mode. An example of the deconvolution results for $\left[\mathrm{C}_{2} \mathrm{mim}\right] \mathrm{CF}_{3} \mathrm{SO}_{3}$ are presented in panel (B). 


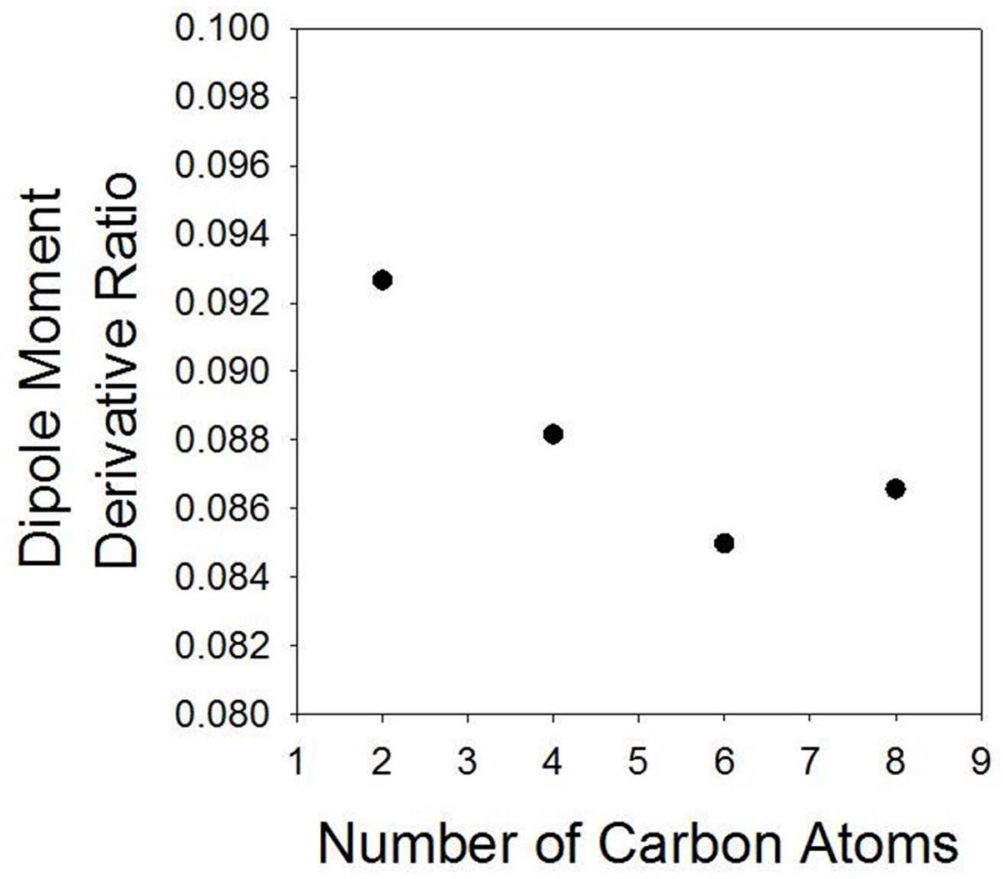

Fig. 2.

A plot of the dipole moment derivative ratio of $\left[\mathrm{C}_{n} \mathrm{mim}\right] \mathrm{CF}_{3} \mathrm{SO}_{3}$ versus the number of carbon atoms composing the alkyl side chain on the imidazolium ring. The temperature is $30^{\circ} \mathrm{C}$ for all of the samples. 


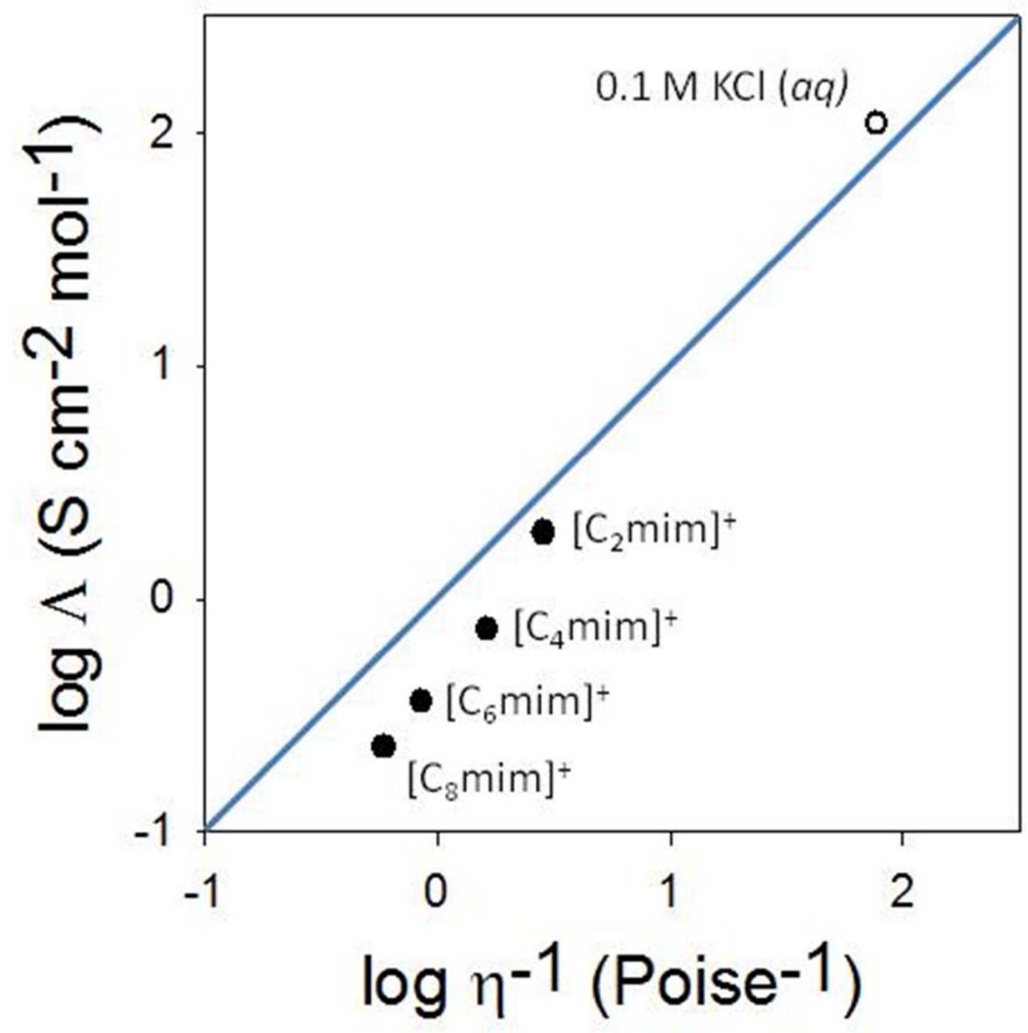

Fig. 3.

A Walden plot for $\left[\mathrm{C}_{n}\right.$ mim $] \mathrm{CF}_{3} \mathrm{SO}_{3}$ is provided to illustrate the relative placement of the ionic liquids relative to the ideal system line (shown as the blue diagonal). The transport properties of $0.1 \mathrm{M} \mathrm{KCl}(a q)$ are included for reference. The temperatures of the ionic liquids are $30^{\circ} \mathrm{C}$. 


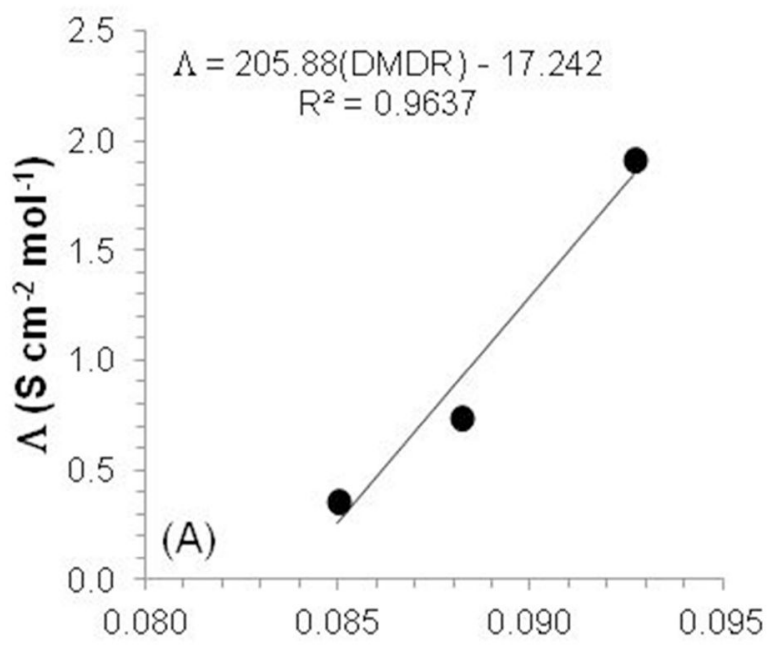

Dipole Moment Derivative Ratio

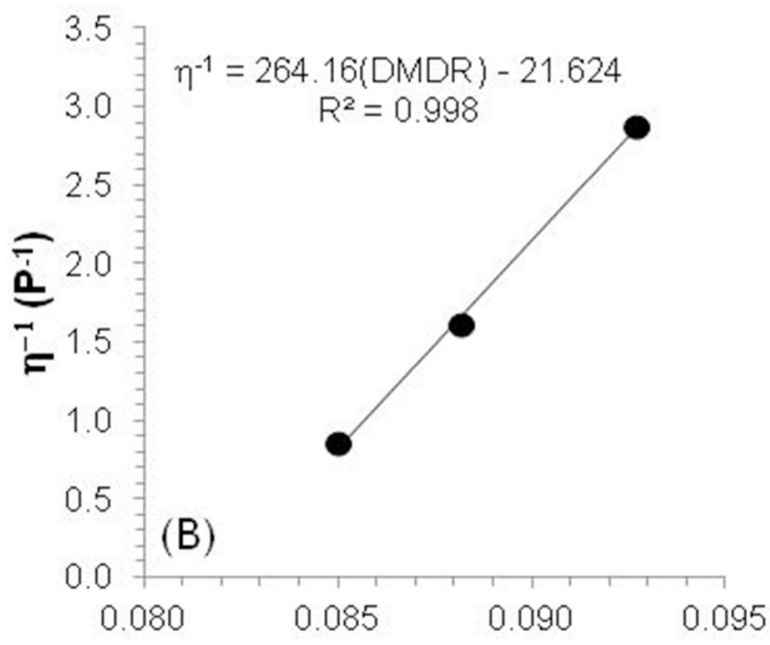

Dipole Moment Derivative Ratio

Fig. 4.

Correlations between (A) molar conductivity and (B) fluidity and the dipole moment derivative ratio (DMDR) of $\left[\mathrm{C}_{n} \mathrm{mim}\right] \mathrm{CF}_{3} \mathrm{SO}_{3}$ with $n=2,4$, and 6 . The temperature is $30^{\circ} \mathrm{C}$. 